\title{
GABA Levels in The Dorsal Anterior Cingulate Cortex Associated with Difficulty lgnoring Smoking-Related Cues in Tobacco-Dependent Volunteers
}

\author{
Amy C Janes*,', John Eric Jensen', Stacey L Farmer', Blaise deB Frederick', Diego A Pizzagalli' and \\ Scott E Lukas'
}

'Brain Imaging Center, McLean Hospital, Harvard Medical School, Belmont, MA, USA

\begin{abstract}
Substance abusers have difficulty ignoring drug-related cues, which is associated with relapse vulnerability. This 'attentional bias' towards drug cues translates into an inability to ignore drug-related stimuli and may reflect deficits in the brain regions, such as the dorsal anterior cingulate cortex (dACC) - a key region in cognitive control and adaptive decision making. Quantifying relationships between attentional biases to drug cues and $\mathrm{dACC}$ neurochemistry could aid in identifying neurobiological mechanisms associated with increased relapse vulnerability precipitated by drug cues. As gamma-aminobutyric acid (GABA) deficits have been linked to impaired cognition and addictive disorders, we hypothesized that reduced GABA in the AACC would be associated with increased attentional biases towards smoking-related cues. We confirmed this hypothesis among nicotine-dependent tobacco smokers by combining an offline behavioral measure of attentional bias with magnetic resonance spectroscopy. Smokers with the greatest attentional bias also experienced more negative affect during early nicotine withdrawal. Findings revealed a relationship between heightened reactivity to drug cues, and both decreasing dACC GABA and early withdrawal symptoms. Because reduced GABA function in frontal brain regions disrupt cognitive function, our findings suggest that smokers with diminished dACC GABA may lack the cognitive resources to successfully ignore highly salient distractors such as tobacco-related stimuli and therefore might be more prone to cue-induced relapse. This newly discovered relationship between DACC GABA and attentional bias provides evidence for a neurochemical target, which may aid smoking cessation in highly cue-reactive individuals.

Neuropsychopharmacology (2013) 38, II I3-1 I20; doi: 10.1038/npp.20 13. 10; published online 6 February 2013
\end{abstract}

Keywords: GABA; dACC; negative affect; attentional bias; tobacco; nicotine dependence

\section{INTRODUCTION}

Dependence on tobacco-derived nicotine continues to be a global health epidemic (World Health Organization, 2008). Unfortunately, most smokers are unable to maintain longterm abstinence (Gonzales et al, 2006; Hughes et al, 2003), highlighting the need to develop more effective treatments and novel approaches for identifying relapse prone individuals. One difficulty in developing effective smoking cessation therapies is that individuals may continue to smoke owing to a range of factors, suggesting that more specialized treatment approaches targeting specific risk factors are needed. Reactivity to smoking cues is one factor associated with cigarette craving, which may precipitate relapse. In fact, probing cue reactivity using a modified version of the Stroop task, which assesses the ability to

\footnotetext{
*Correspondence: Dr AC Janes, Brain Imaging Center, Mail Stop 310 , McLean Hospital, Harvard Medical School, II 5 Mill Street Belmont, MA 02478, USA. Tel: (617) 855 3244, Fax: (617) 855 2770, E-mail: ajanes@mclean.harvard.edu

Received I November 2012; revised 2 January 2013; accepted 3 January 2013; accepted article preview online 10 January 2013
}

perform a primary task within the context of distracting smoking-related cues, has shown promise for predicting smoking cessation outcomes (Waters et al, 2003; Janes et al, 2010a).

Successful performance on the smoking emotional Stroop (SES) task requires that participants ignore smoking-related cues in favor of task elements unrelated to smoking. The inability to efficiently ignore drug-related stimuli (ie, an attentional bias towards drug cues) may reflect deficits in frontal brain regions critically implicated in cognitive control and decision making, which may contribute to the persistence of drug addiction (Goldstein and Volkow, 2011). In particular, the dorsal anterior cingulate cortex (dACC) has a key role in addiction as this region is not only implicated in smoking cue attentional bias (Luijten et al, 2012), but also reward-based decision making, conflict monitoring (Botvinick, 2007; Bush et al, 2002), and cognitive regulation of smoking cue-induced craving (Brody et al, 2007). In line with this conjecture, the dACC has been found to be dysfunctional in more heavily nicotine-dependent smokers (Hong et al, 2009) as well as those likely to relapse (Janes et al, 2010a). Collectively, these 
studies suggest that therapies targeted at ameliorating dACC dysfunction may reduce attentional bias and, as a result, minimize relapse risk. However, neurochemical disruptions within the dACC have yet to be associated with attentional bias for drug cues.

In the present study, we focused on gamma-aminobutyric acid (GABA) concentrations in the dACC, as reduced prefrontal GABA has been associated with impaired cognition (Enomoto et al, 2011; Fujiwara et al, 2011; Paine et al, 2011) and addictive disorders (Behar et al, 1999; $\mathrm{Ke}$ et al, 2004; Levy and Degnan, 2012). Additionally, GABAergic agonists reduce cigarette consumption in clinical trials (Franklin et al, 2009), and disrupt reactivity to nicotine cues in preclinical studies (Paterson et al, 2005). Specifically, we assessed the relationship between attentional bias towards smoking-related words using the SES task and dACC GABA concentrations using proton magnetic resonance spectroscopy $\left({ }^{1} \mathrm{H}-\mathrm{MRS}\right)$ in nicotine dependent smokers. To test whether attentional bias was associated with early nicotine withdrawal symptoms, we also evaluated the relationship between attentional bias and changes in mood and craving due to short-term nicotine abstinence. Our findings provide novel insights into the neurobiological substrates of attentional bias, and may offer early evidence that GABA may represent a neurochemical target for treating cue-reactive smokers.

\section{MTERIALS AND METHODS}

\section{Study Participants}

Fifteen nicotine-dependent tobacco smokers (eight women) between the ages of 18-33, who did not participate in our previous studies (Janes et al, 2010a,b), underwent neuroimaging and behavioral testing at the McLean Imaging Center of McLean Hospital. Participants reported smoking $\geqslant 10$ cigarettes/day over the past 6 months, and were moderately to heavily nicotine dependent as measured by the Fagerstrom test for nicotine dependence (FTND; Fagerstrom, 1978). Participants were assessed by the Structured Clinical Interview for DSM and met criteria for current nicotine dependence. Participants were excluded if they had a lifetime diagnosis of the following conditions: organic mental disorder, bipolar or unipolar depression, and schizophrenia spectrum disorder. Participants were excluded for current substance use disorder other than nicotine dependence. Smokers also were excluded for pregnancy, current psychotropic drug use, recent drug use, or excessive alcohol use. Subjects were recruited using online advertisements and fliers posted in the Boston area. All participants provided written informed consent before participating in any study procedures, and the institutional review board at McLean Hospital approved this study.

\section{Participant Assessments}

Lifetime tobacco use was assessed by pack-years, while recent smoking was measured by expired carbon monoxide (CO; Micro Smokerlyzer II, Bedfont Scientific Instruments). Cigarette craving was assessed by the Tiffany Questionnaire of Smoking Urges (QSU; Tiffany and Drobes, 1991), whereas the Barratt Impulsiveness Scale (BIS; Patton et al,
1995) was used to measure impulsivity. State affect also was measured using the Positive and Negative Affect Schedule (PANAS; Watson et al, 1988). To standardize the time since a cigarette was last smoked, all participants smoked one of their own cigarettes at the beginning of the study. MRS was measured $\sim 2.5 \mathrm{~h}$ after smoking while the Stroop task was administered $\sim 4.5 \mathrm{~h}$ after smoking. To assess the onset of withdrawal-induced craving and negative mood, both the QSU and PANAS were administered $\sim 1 \mathrm{~h}$ after smoking and again $\sim 3.5 \mathrm{~h}$ after smoking. Specifically, we focused on increases in negative affect using the negative subscales of the PANAS (Watson et al, 1988). Changes in craving and negative affect were calculated by comparing responses made at 3.5 vs $1 \mathrm{~h}$ post smoking.

To rule out the possibility that sex differences might be driving any effects, all measures were compared between men and women using two-tailed $t$-tests. Additionally, menstrual cycle phase was obtained in women using selfreport, and all measures were compared between women in the luteal $v s$ follicular menstrual cycle phase.

\section{Magnetic Resonance Spectroscopy}

Data acquisition. MRS data were collected on a 3T Siemens TRIO Tim, whole-body, clinical MR system (Erlangen, Germany) using a 32-channel phased-array design RF head coil operating at $123 \mathrm{MHz}$ for proton imaging and spectroscopy. High-resolution T1-weighted anatomical images were used to position a single $2 \times 2 \times 3$ $\mathrm{cm}$ voxel in the dACC (Figure 1). Proton MRS used a modified J-resolved PRESS protocol (two-dimensional (2D)-JPRESS). Shimming of the magnetic field within the prescribed voxel was done automatically using an automated shimming routine. Following the additional automated optimization of water suppression power, carrier frequency, tip angles, and coil tuning, the 2D-JPRESS sequence collected 22 echo time (TE)-stepped spectra with the $\mathrm{TE}$ ranging from 30 to $350 \mathrm{~ms}$ in $15 \mathrm{~ms}$ increments. Acquisition parameters were: repetition time $=2 \mathrm{~s}, \mathrm{f} 1$ acquisition bandwidth $=67 \mathrm{~Hz}$, spectral bandwidth $=2 \mathrm{kHz}$, readout duration $=512 \mathrm{~ms}, \mathrm{NEX}=16 / \mathrm{TE}$-step, total scan duration $=12 \mathrm{~min}$.

Proton MRS processing/analysis. All spectroscopic data processing and analysis was undertaken on a LINUX

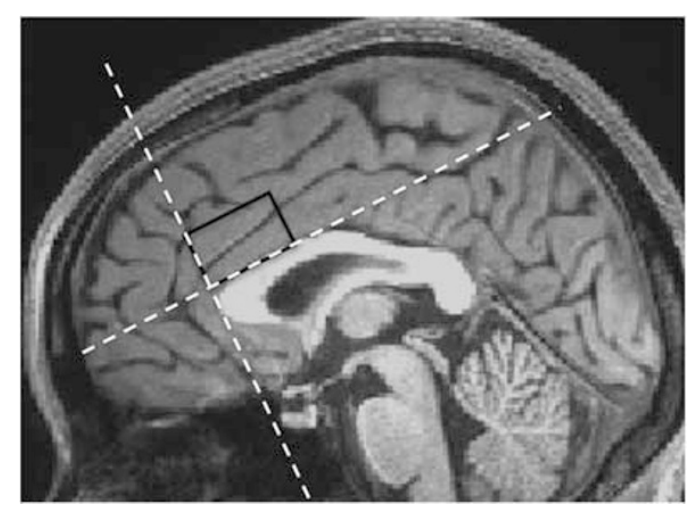

Figure I Sagittal view of a representative $T_{1}$-weighted image illustrating $2 \times 2 \times 3 \mathrm{~cm}$ voxel placement in the $\mathrm{dACC}$. 
workstation. In order to quantify GABA with the JPRESS data, the 22 TE-stepped free-induction decay was first zerofilled out to 64 points, Gaussian-filtered, and Fourier transformed. Consistent with our validated methods, every J-resolved spectral extraction within a bandwidth of $67 \mathrm{~Hz}$ was fitted with LCModel and its theoretically-correct template, which used an optimized GAMMA-simulated J-resolved basis sets modeled for $2.89 \mathrm{~T}$ (Friedman et al, 2012; Henry et al, 2011; Jensen et al, 2009). The integrated area under the entire 2D surface for each metabolite was calculated by summing the raw peak areas across all 64 J-resolved extractions for each metabolite. GABA metabolites were expressed as ratios to total creatine $(\mathrm{Cr})$. A representative spectrum is shown in Figure 2.

\section{Image Segmentation/voxel Tissue Analysis}

All image segmentation was performed using FSL version 4.1.1 (FMRIB Software Library; Analysis Group, FMRIB; Oxford, UK) in a VMware virtual machine running on a PC. For voxel tissue partial-volume estimation, the highresolution $\mathrm{T}_{1}$-weighted axial images were first automatically segmented into cortical gray matter (GM), white matter (WM), and cerebral spinal fluid (CSF) compartments using the segmentation tool in the software package FSL 4.1.1. This package allows for optimized and fully automated segmenting of both cortical tissue ('FAST'-FMRIB Software Library; Analysis Group, FMRIB; Oxford, UK) as well as sub-cortical tissue ('FIRST'-FMRIB Software Library; Analysis Group, FMRIB; Oxford, UK). The segmented

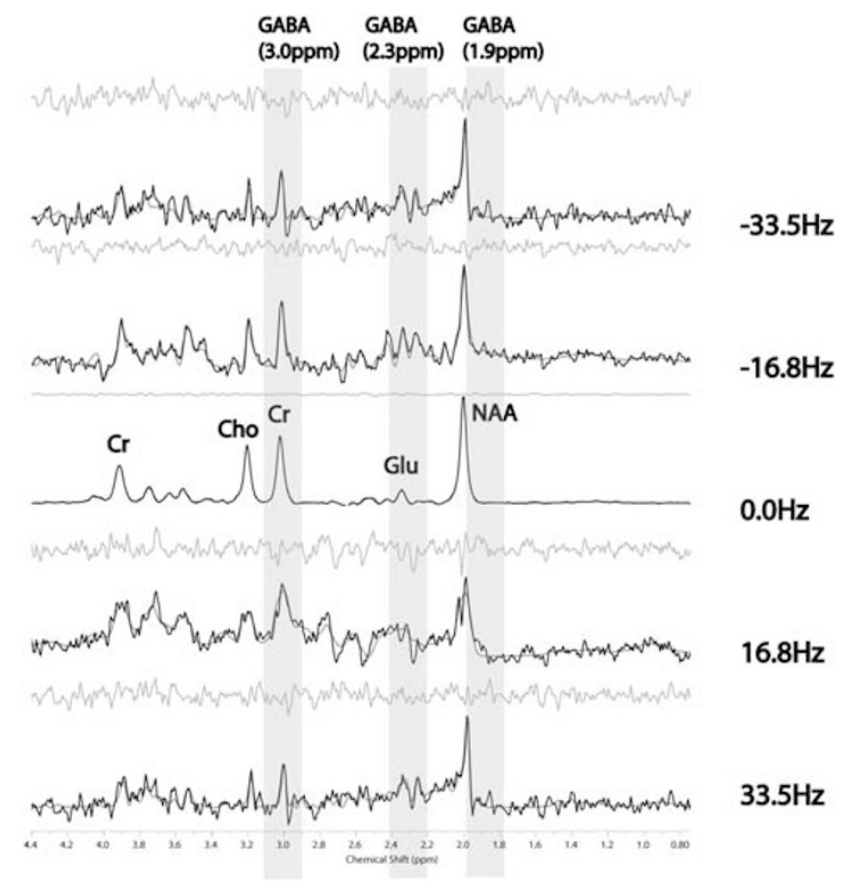

Figure 2 Selected J-resolved spectral extractions across the $67 \mathrm{~Hz}$ bandwidth in the J-resolved 2D spectrum, displayed with I Hz exponential time-domain filtering and LCModel fits. Displayed spectral extractions are a subset of the 64J-resolved spectra from, which GABA is derived using LCModel. Each spectral extraction is modeled with its specific theoretical J-resolved LCModel template and the output from each extraction integrated across all 64 extractions to derive the final metabolite areas. images were then reformatted for input into an automated voxel coregistration and partial-volume analysis in-house program written in C-code. Subsequently, the volumetric tissue contribution for each oblique dACC voxel was determined and volumetric contributions of total GM, WM, and CSF calculated.

\section{Smoking Emotional Stroop}

Participants performed a behavioral computerized SES task outside of the magnet $\sim 4.5 \mathrm{~h}$ after smoking and $2 \mathrm{~h}$ after MRS scanning. The SES task displayed neutral and smoking associated words, matched for length, and use frequency in the English language, in red, green, or blue font using similar procedures as implemented by others (Gross et al, 1993; Munafo et al, 2003; Waters et al, 2003; Wetherill et al, 2012) and as in our previously published work (Janes et al, 2010a,b). Participants were instructed to press a button to identify font color as accurately and quickly as possible while ignoring word meaning. Participants first completed a 96-trial practice block of letter strings followed by two experimental blocks containing 33 trials (words) each. The neutral block preceded the smoking block by a $5 \mathrm{~s}$ break and in each block, every word $(n=11)$ was repeated three times (once for each color). Each trial began with a $500 \mathrm{~ms}$ fixation cross, followed by a word presentation, and then a $500 \mathrm{~ms}$ intertrial interval. Words were presented until a response was made, or disappeared after $3 \mathrm{~s}$ if no response was made. Eprime software (Psychology Software Tools, Pittsburgh, PA) was used to present stimuli and record responses. A two step procedure was implemented to minimize outlier response: trials with reaction times (RT) $<150 \mathrm{~ms}$ and $>1500 \mathrm{~ms}$ were first excluded; next, trials with natural log-transformed RT falling out of the range of mean \pm three SD (calculated for each participant individually after the removal of trials with $150 \mathrm{~ms}>\mathrm{RT}>1500 \mathrm{~ms}$ ) were also excluded (on average, $4.5 \%$ of the trials were excluded). Following established procedures (eg, Janes et al, 2010a,b; Waters et al, 2003), an attentional bias score was computed as $\mathrm{RT}_{\text {smoking }}-\mathrm{RT}_{\text {neutral }}$ for trials with correct responses. Higher values indicate enhanced interference effects associated with smoking-related words.

\section{Correlation Analyses}

A Pearson's correlation coefficient was calculated between smoking attentional bias $\left(\mathrm{RT}_{\text {smoking }}-\mathrm{RT}_{\text {neutral }}\right)$ and $\mathrm{dACC}$ $\mathrm{GABA} / \mathrm{Cr}$ concentration. To determine whether attentional bias and dACC GABA/Cr levels were associated with changes in mood and craving over short-term nicotine abstinence, correlation coefficients were also calculated between attentional bias, dACC GABA/Cr and changes in QSU and negative affect PANAS scores $3.5 v s 1 \mathrm{~h}$ after cigarette smoking. Finally, to identify possible relationships between demographic factors and our variables of interest, Pearson's correlation coefficients were also calculated between attentional bias, dACC GABA/Cr, and: age, FTND, expired CO, pack-year, and BIS. To ensure that findings were not unduly affected by possible outliers, correlations were confirmed using Spearman's nonparametric test. 


\section{RESULTS}

\section{Participant Demographics}

On average, participants were $25.5 \pm 4.8$ (SD) years old, with $15.5 \pm 2.2$ years of education. Participants had an average FTND score of $6.2 \pm 1.1$, indicating moderate to heavy nicotine dependence. Participants also reported smoking $15.5 \pm 3.8$ cigarettes/day, and had an average pack-year of $6.7 \pm 4.9$ years. Just before MRS procedures, participants had an expired $\mathrm{CO}$ of $27.7 \pm 12.9 \mathrm{ppm}$. The average change in negative affect as measured by the PANAS was $0.33 \pm 1.63$. Over the course of the experiment, cigarette craving significantly increased $\left(P<0.01, t_{14}=3.1\right)$ as measured by the QSU.

\section{SES Main Effect}

During the SES task, smokers took an average of $35.38 \pm 76.91 \mathrm{~ms}$ longer to respond to smoking $v s$. neutral words. Although this difference between smoking and neutral word RT was not statistically significant (likely due to large individual differences), 11 of the 15 participants showed a Stroop interference effect greater than zero (ie, some degree of smoking-related interference). A binomial distribution test indicated that this effect was significant (binomial $\left.p_{(11 / 15)}=0.042\right)$.

\section{Behavioral and Neurochemical Associations}

As hypothesized, a significant negative correlation emerged between attentional bias towards smoking cues and dACC GABA/Cr levels $(r=-0.63, P=0.011 ; 95 \%$ confidence interval (CI): -0.86 to -0.18 ; Spearman's rho $=-0.68$, $P<0.01$; Figure 3). Analyses were repeated controlling for age and expired $\mathrm{CO}$, as we found relationships between $\mathrm{dACC} \mathrm{GABA} / \mathrm{Cr}$ and age $(r=-0.54, P=0.04 ; 95 \% \mathrm{CI}$ : -0.82 to -0.037$)$ and $\mathrm{GABA} / \mathrm{Cr}$, and expired $\mathrm{CO}$ $(r=-0.51, P=0.05 ; 95 \% \mathrm{CI}:-0.81$ to -0.002$)$. When controlling for age and $\mathrm{CO}$ using a partial correlation, the association between dACC GABA and smoking word

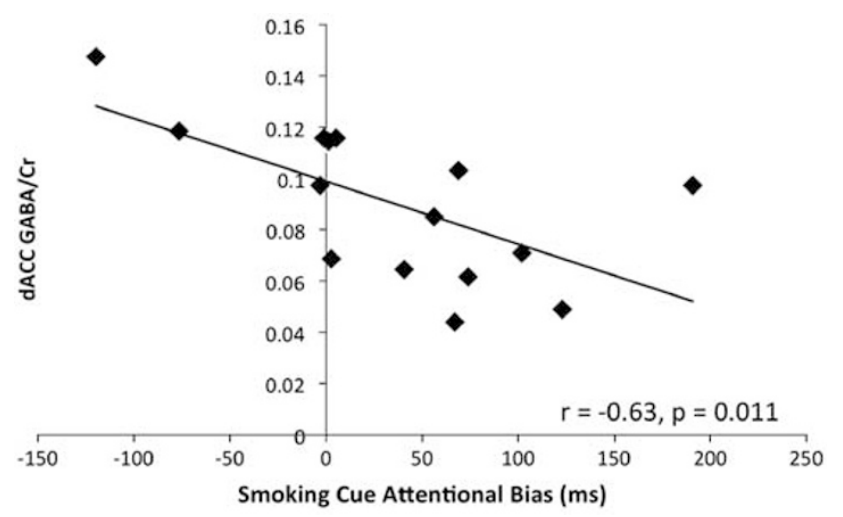

Figure 3 Correlation between smoking cue attentional bias and dACC $\mathrm{GABA} / \mathrm{Cr}$. The $x$ axis represents smoking cue attentional bias, which was calculated by $R T_{\text {smoking }}-R T_{\text {neutral }}(\mathrm{ms})$, and the $y$ axis shows the $d A C C$ concentration of GABA/Cr. Smokers with the greatest smoking cue attentional bias also had the lowest levels of dACC GABA/Cr $(r=-0.63$, $P=0.011)$. attentional bias remained (two-tailed Pearson's $r=-0.61$, $P=0.026)$.

A significant positive correlation emerged between enhanced attentional bias to smoking cues and increased negative affect over the course of the experiment, as measured by the PANAS $(r=0.77, P<0.001 ; 95 \%$ CI: $0.42-0.92$; Spearman's rho $=0.82, P<0.001$; Figure 4$)$. Thus, attentional biases toward smoking-related cues increased with increasing levels of negative affect over the course of the experiment. In addition, a trend for a negative correlation emerged between GABA and increased negative affect $(r=-0.46, P=0.08 ; 95 \%$ CI: -0.79 to 0.07$)$. There was no relationship between FTND, BIS, pack-year, or QSUmeasured craving and attentional bias or dACC GABA/Cr levels. All associations with dACC GABA/Cr levels were unrelated to $\mathrm{Cr}$, which was the internal reference for each participant.

\section{Sex Differences}

No differences were found between men and women on any measure. Of the eight women studied, four were in the luteal phase and four were in the follicular phase of their menstrual cycle. No differences were found between women in different phases of their menstrual cycle.

\section{Voxel Tissue}

On average, the dACC voxel was comprised of $58.8 \pm 5.7$ percent GM, $30.8 \pm 4.5$ percent $\mathrm{WM}$, and $10.44 \pm 3.9$ percent CSF. We found no significant correlation between GABA levels and the amount of GM, WM, and CSF in the defined voxel.

\section{DISCUSSION}

In addition to finding a significant percentage of participants with an attentional bias toward smoking cues, we report a negative correlation between attentional bias and dACC GABA/Cr concentration. Our finding that decreasing

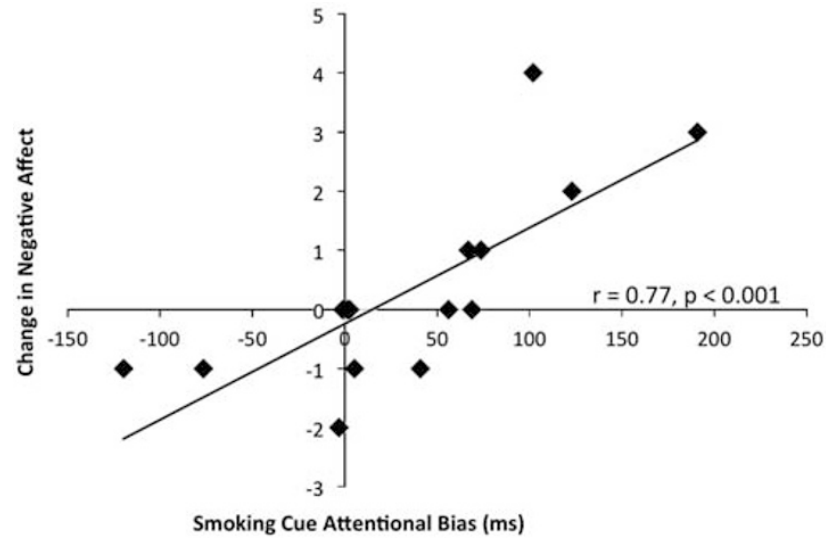

Figure 4 The $x$ axis represents smoking cue attentional bias, which was calculated by $R T_{\text {smoking }}-R T_{\text {neutral }}(\mathrm{ms})$, and the $y$ axis shows the change in negative affect as measured by the PANAS $3.5 \mathrm{vs} \mathrm{I} \mathrm{h} \mathrm{post} \mathrm{smoking.}$ Smokers with the greatest smoking cue attentional bias also experienced the greatest increase in negative affect over the course of the experiment $(r=0.77, P<0.00 I)$. 
dACC GABA levels were associated with poorer SES performance supports the concept that the dACC has a critical role in attentional biases toward smoking-related cues (Luijten et $a l, 2011$ ) and cognitive control in general (Botvinick, 2007; Bush et al, 2002). During tasks requiring cognitive effort, such as those containing distracting stimuli, the dACC along with other prefrontal brain regions is critically implicated in focusing attention away from the distractors and sustaining task-related goals (Botvinick, 2007; Bush et al, 2000; Bush and Shin, 2006; Medalla and Barbas, 2009). During the SES task, intact and efficient dACC function may be necessary for smokers to ignore the irrelevant smoking stimuli and attend to information necessary to perform the primary task. Disruptions in this system may therefore lead to interference by smoking stimuli.

Reductions in dACC GABA/Cr may contribute to smoking cue attentional bias as mounting evidence indicates that low levels of prefrontal GABA contribute to attentional and cognitive dysfunction at a more basic level (Enomoto et al, 2011; Fujiwara et al, 2011; Paine et al, 2011; Pehrson et al, 2013). On a cellular level, GABA enhances neuronal communication by inhibiting inappropriate activation or 'noise' while allowing selective activation of task-relevant signals, thus facilitating optimal cognition (Paine et al, 2011; Rolls et al, 2008; Winterer and Weinberger, 2004). Although reduced prefrontal GABA is thought to partly explain cognitive deficits in schizophrenia (Enomoto et al, 2011; Paine et al, 2011; Rolls et al, 2008) and age-related cognitive decline (Fujiwara et al, 2011), the current findings suggest that lower levels of prefrontal GABA may account for cognitive interference by smoking cues in otherwise healthy, young smokers. Specifically, lower dACC GABA may reduce the ability to filter irrelevant information leading to distraction by salient stimuli such as smoking cues.

Similar to attentional bias, negative mood has been associated with smoking relapse vulnerability (Shiffman and Waters, 2004), suggesting that these factors may be important targets for smoking cessation treatments. Consistent with previous findings (Bradley et al, 2007), we uncovered a strong positive relationship between negative mood and smoking attentional bias, implying that these two relapse vulnerability risk factors may be linked. In fact, negative affect generally disrupts cognitive performance during interference tasks (Sommer et al, 2008), indicating that a more global association exists between affect and cognitive interference. Not only do negative emotions lead to greater interference measured behaviorally, but also negative affect reduces dACC activation during interference processing (Sommer et al, 2008). One hypothesis is that the dACC may be the neurobiological link between negative affect and cognition (Shackman et al, 2011; Sommer et al, 2008). Therefore, smokers experiencing negative affect may have greater difficulty ignoring smoking cues owing to the influence of negative mood on dACC function. When smokers with relatively lower levels of dACC GABA experience negative affect, this may tax an already dysfunctional dACC, making it even more difficult to ignore salient distractors such as smoking cues. We also found a trend negative association between dACC GABA/Cr and negative mood. Given the relationship between reduced brain GABA concentrations and mood disorders (Brambilla et al, 2003; Petty, 1995), it is possible that a lower dACC GABA concentration may enhance the likelihood of experiencing nicotine withdrawal-induced negative affect. Although plausible, this proposed relationship between dACC GABA and nicotine withdrawal requires confirmation in a larger sample of smokers.

Although the relationship between decreased cortical GABA and substance abuse has been previously established (Ke et al, 2004; Behar et al, 1999, Levy and Degnan, 2012), this is the first reported association between dACC GABA/ $\mathrm{Cr}$ concentration and smoking attentional bias. GABAbased therapies have been explored for numerous addictive disorders (eg, Brebner et al, 2002; Brodie et al, 2003; Franklin et al, 2009; Kampman et al, 2004; Kaplan et al, 2003) and have been found to reduce cigarette consumption (Franklin et al, 2009) and cigarette craving (Sofuoglu et al, 2005). Preclinically, GABAergic drugs improve drug-induced cognitive disruptions (Arai et al, 2009; Porrino et al, 2012) and reduce responding to nicotine cues (Paterson et al, 2005). Additionally, GABAergic drugs enhance traditional Stroop performance in clinical studies (Sofuoglu et al, 2005). These studies suggest that pharmacological enhancement of GABAergic function may not only reduce smoking, but may buffer against future relapses by improving cognitive deficits such as those contributing to smoking attentional bias.

Cigarette craving increased over the course of the experiment, yet we did not find an association between craving and attentional bias, which is consistent with a meta-analysis showing that the relationship between tobacco craving and attentional bias is weak, especially when using behavioral measures such as the SES (Field et al, 2009). Also consistent with previous research, we found no influence of sex on attentional bias and nonabstinent negative affect (Leventhal et al, 2007). Nevertheless, while some brain regions experience GABA fluctuations due to menstrual cycle phase, ACC GABA levels are unaffected by the menstrual cycle (Harada et al, 2011). Additionally, smoking is thought to abolish the influence of the menstrual cycle on occipital cortex GABA fluctuations such that women smokers do not experience a rise in GABA during the follicular phase (Epperson et al, 2005). On the basis of these past studies, menstrual cycle influences on our results were unlikely. However, given our small sample size and the variability of self-reported menstrual cycle phase information, the influence of sex on the relationship between dACC GABA/Cr and attentional bias needs to be tested more directly.

\section{LIMITATIONS}

Despite the wealth of research suggesting prefrontal GABA has a role in cognition (Enomoto et al, 2011; Fujiwara et al, 2011; Paine et al, 2011; Pehrson et al, 2013; Rolls et al, 2008; Winterer and Weinberger, 2004), our results are correlative and thus we are unable to determine whether decreased dACC GABA/Cr directly causes enhanced attentional bias. Furthermore, we have not directly confirmed that GABA levels are stable during the time between the MRS scan and the Stroop task. However, past work suggests that cortical 
GABA levels do not change following $48 \mathrm{~h}$ of nicotine abstinence (Epperson et al, 2005), suggesting that it is unlikely that GABA levels would have changed dramatically over the $2 \mathrm{~h}$ between the MRS scan and Stroop task performance. The stability of GABA following $48 \mathrm{~h}$ of abstinence also suggests that the individual variability in GABA reported here was not due to early nicotine withdrawal, but may be a pre-existing condition. While this interpretation is plausible based on the prior literature, it needs to be tested empirically.

In the present study, the participant population was restricted to relatively young, healthy smokers with moderate to high levels of nicotine dependence. Our participants were also well educated (having received an average of $15.5 \pm 2.2$ years of schooling) and showed no influence of trait impulsivity on attentional bias, negative affect, and GABA levels. Therefore, we are unable to generalize the association between GABA and attentional bias to more global cognitive disruptions in this population. Additionally, we found no correlations between GABA levels and specific tissue concentrations in the dACC voxel, suggesting the relationship between GABA and attentional bias to smoking cues is due to individual differences in GABA levels rather than tissue differences within the dACC voxel. However, the relatively limited sample size also suggests that the current finding should be interpreted cautiously. However, future research involving a larger, more diverse sample of smokers may help clarify some of these issues.

As our population consisted entirely of smokers, we cannot conclude that smokers with enhanced attentional bias have a decrease in GABA relative to normative values. However, given the wealth of literature associating lower GABA levels with disrupted cognition and addictive disorders, it is possible that highly cue-reactive smokers have reduced GABA levels. Finally, MRS also limits our results as this method cannot pinpoint the cause of reduced GABA concentrations, nor can it distinguish between separate pools of GABA (eg, intra- vs extracellular). Future preclinical work may be useful in clarifying the direct cause of dACC GABA associations with attentional bias.

\section{CONCLUSION}

Despite these limitations, the relationship between decreasing $\mathrm{dACC} \mathrm{GABA} / \mathrm{Cr}$ and increasing attentional bias suggests that GABAergic treatments may be helpful for the subset of smokers who are more distracted by smoking cues. Within our sample, there was a range of Stroop RT scores, emphasizing that smokers are not homogeneous in their reactivity to smoking stimuli. This variability is consistent with past work showing that relapse vulnerable smokers have significantly greater attentional bias to smoking cues than smokers able to maintain abstinence (Janes et al, 2010a; Waters et al, 2003). This sample of studied smokers also included those who more easily ignored smoking vs neutral words. Quicker response times to smoking vs neutral words has been shown in nonabstinent smokers, as they may not be as preoccupied by smoking cues as abstinent smokers (Gross et al, 1993). Our work extends this finding by suggesting that smokers vary in their level of attentional bias, which is associated with dACC GABA levels. Accordingly, therapies that address a possible GABAergic deficit may reduce smoking cue attentional bias and enhance cessation success in cue-reactive smokers. Future research should determine whether our finding is unique to nicotine dependence or whether reduced GABA levels contribute to enhanced attentional bias to drug stimuli across addictive disorders. As attentional bias for drug cues is predictive of treatment outcomes across many addictive disorders including nicotine, alcohol, and heroin (Fadardi and Cox, 2008; Janes et al, 2010a; Marissen et al, 2006; Waters et al, 2003), therapeutics targeting the GABA system may be successful relapse prevention aids for individuals abusing a variety of substances.

\section{ACKNOWLEDGEMENTS}

This work was supported by the National Institute on Drug Abuse Grant number K01DA029645.

\section{DISCLOSURE}

ACJ, JEJ, SLF, BdeBF, and SEL declare no conflict of interest. DAP has received consulting fees from AstraZeneca, Ono Pharma USA, Shire, and Johnson and Johnson for projects unrelated to the current research.

\section{REFERENCES}

Arai S, Takuma K, Mizoguchi H, Ibi D, Nagai T, Kamei $\mathrm{H}$ et al (2009). GABAB receptor agonist baclofen improves methamphetamine-induced cognitive deficit in mice. Eur J Pharmacol 602: 101-104.

Behar KL, Rothman DL, Petersen KL, Hooten M, Delany R, Petroff OAC et al (1999). Preliminary evidence of low cortical GABA levels in localized 1H-MR spectra of alcohol-dependent and hepatic encephalopathy patients. Am J Psychiatry 156: 952-954.

Botvinick MM (2007). Conflict monitoring and decision making: Reconciling two perspectives on anterior cingulate function. Cogn Affect Behav Neurosci 7: 356-366.

Bradley BP, Garner M, Hudson L, Mogg K (2007). Influence of negative affect on selective attention to smoking-related cues and urge to smoke in cigarette smokers. Behav Pharmacol 18: 255-263.

Brambilla P, Perez J, Barale F, Schettini G, Soares JC (2003). GABAergic dysfunction in mood disorders. Mol Psychiatry 8: 721-737.

Brebner KM, Childress AR, Roberts CS (2002). A potential role for GABA(B) agonists in the treatment of psychostimulant addiction. Alcohol Alcohol 37: 478-484.

Brody AL, Mandelkern MA, Olmstead RE, Jou J, Tiongson E, Allen V (2007). Neural substrates of resisting craving during cigarette cue exposure. Biol Psychiatry 62: 642-651.

Brodie JD, Figueroa E, Dewey SL (2003). Treating cocaine addiction: From preclinical to clinical trial experience with gamma-vinyl GABA. Synapse 50: 261-265.

Bush G, Luu P, Posner MI (2000). Cognitive and emotional influences in anterior cingulate cortex. Trends Cogn Sci 4: 215-222.

Bush G, Shin LM (2006). The multi-source interference task: an fMRI task that reliably activates the cingulo-frontal-parietal cognitive/attention network. Nat Protoc 1: 308-313. 
Bush G, Vogt BA, Holmes J, Dale AM, Greve D, Jenike MA et al (2002). Dorsal anterior cingulate cortex: a role in reward-based decision making. Proc Natl Acad Sci USA 99: 523-528.

Enomoto T, Tse MT, Floresco SB (2011). Reducing prefrontal gamma-aminobutyric acid activity induces cognitive, behavioral, and dopaminergic abnormalities that resemble schizophrenia. Biol Psychiatry 69: 432-441.

Epperson CN, O’Malley S, Czarkowski KA, Gueorguieva R, Jatlow P, Sanacora G et al (2005). Sex, GABA, and Nicotine: the impact of smoking on cortical GABA levels across the menstrual cycle as measured with proton magnetic resonance spectroscopy. Biol Psychiatry 57: 44-48.

Fadardi JS, Cox WM (2008). Alcohol-attentional bias and motivational structure as independent predictors of social drinkers' alcohol consumption. Drug Alcohol Depend 97: 247-256.

Fagerstrom KO (1978). Measuring degree of physical dependence to tobacco smoking with reference to individualization of treatment. Addict Behav 3: 235-241.

Franklin TR, Harper D, Kampman K, Kildea-McCrea S, Jens W, Lynch KG (2009). The GABA B agonist baclofen reduces cigarette consumption in a preliminary double-blind placebocontrolled smoking reduction study. Drug Alcohol Depend 103: $30-36$.

Field M, Munafo MR, Franken IHA (2009). A meta-analytic investigation of the relationship between attentional bias and subjective craving in substance abuse. Psychol Bull 135: 589-607.

Friedman SD, Baker LD, Borson S, Jensen JE, Barsness SM, Craft S et al (2012). Growth hormone-releasing hormone increases brain gaba levels in mild cognitive impairment and healthy aging. Arch Neurol (in press).

Fujiwara H, Zheng M, Miyamoto A, Hoshino O (2011). Insufficient augmentation of ambient GABA responsible for age-related cognitive deficit. Cogn Process 12: 151-159.

Goldstein RZ, Volkow ND (2011). Dysfunction of the prefrontal cortex in addiction: neuroimaging findings and clinical implications. Nat Rev Neurosci 12: 652-669.

Gonzales D, Rennard SI, Nides M, Oncken C, Azoulay S, Billing CB et al (2006). Varenicline, an $\alpha 4 \beta 2$ nicotinic acetylcholine receptor partial agonist, vs sustained-release bupropion and placebo for smoking cessation. A randomized controlled trial. JAMA 296: 47-55.

Gross TM, Jarvik ME, Rosenblatt MR (1993). Nicotine abstinence produces content-specific Stroop interference. Psychopharmacology (Berl) 110: 333-336.

Harada M, Kubo H, Nose A, Nishitani H, Matsuda T (2011). Measurement of variation in the human cerebral GABA level in vivo MEGA-editing proton MR spectroscopy using a clinical $3 \mathrm{~T}$ instrument and its dependence on brain region and the female menstrual cycle. Hum Brain Mapp 32: 828-833.

Henry ME, Lauriat TL, Shanahan M, Renshaw PF, Jensen JE (2011). Accuracy and stability of measuring GABA, glutamate, and glutamine by proton magnetic resonance spectroscopy: a phantom study at 4 tesla. J Magn Reson 208: 210-218.

Hong LE, Gu H, Yang Y, Ross TJ, Salmeron BJ, Buchholz B et al (2009). Association of nicotine addiction and nioctine's actions with separate cingulate cortex functional circuits. Arch Gen Psychiatry 66: 431-441.

Hughes JR, Siffman S, Callas P, Zhang J (2003). A meta-analysis of the efficacy of over-the-counter nicotine replacement. Tob Control 12: 21-27.

Janes AC, Pizzagalli DA, Richardt S, BdeB Frederick, Chuzi S, Pachas $\mathrm{G}$ et al (2010a). Brain reactivity to smoking cues prior to smoking cessation predicts ability to maintain tobacco abstinence. Biol Psychiatry 67: 722-729.

Janes AC, Pizzagalli DA, Richardt S, BdeB Frederick, Holmes AV, Sousa J et al (2010b). Neural substrates of attentional bias for smoking-related cues: An fMRI study. Neuropsychopharmacology 35: 2339-2345.

Jensen JE, Licata SC, Öngür D, Friedman SD, Prescot AP, Henry ME et al (2009). Quantification of J-resolved Proton Spectra in Two-Dimensions with LCModel Using GAMMA-Simulated Basis Sets at 4 Tesla. NMR Biomed 22: 762-769.

Kampman KM, Pettinati H, Lynch KG, Dackis C, Sparkman T, Weigley C et al (2004). A pilot trial of topiramate for the treatment of cocaine dependence. Drug Alcohol Depend 75: 233-240.

Kaplan GB, Leite-Morris KA, Joshi M, Shoeb MH, Carey RJ (2003). Baclofen inhibits opiate-induced conditioned place preference and associated induction of Fos in cortical and limbic regions. Brain Res 987: 122-125.

Ke Y, Streeter CC, Nassar LE, Sarid-Segal O, Hennen J, YurgelunTodd DA et al (2004). Frontal lobe GABA levels in cocaine dependence: a two-dimensional, J-resolved magnetic resonance spectroscopy study. Psychiatry Res 130: 283-293.

Leventhal AM, Boyd S, Moolchan ET, Waters AJ, Lerman C, Pickworth WB (2007). Gender differences in acute tobacco withdrawal: Effects on subjective, cognitive, and physiological measures. Exp Clin Psychopharmacol 15: 21-26.

Levy LM, Degnan AJ (2012). GABA-based evaluation of neurologic conditions: MR spectroscopy. AJNR AM J Neuroradiol (e-pub ahead of print).

Luijten M, Veltman DJ, van den Brink W, Hester R, Field M, Smits $M$ et al (2011). Neurobiological substrate of smoking-related attentional bias. Neuroimage 45: 2374-2381.

Marissen MAE, Franken IHA, Waters AJ, Blanken P, van den Brink W, Hendriks VM (2006). Attentional bias predicts heroin relapse following treatment. Addiction 101: 1306-1312.

Medalla M, Barbas H (2009). Synapses with inhibitory neurons differentiate anterior cingulate from dorsolateral prefrontal pathways associated with cognitive control. Neuron 61: 609-620.

Munafo M, Mogg K, Roberts S, Bradley BP, Murphy M (2003). Selective processing of smoking-related cues in current smokers, ex-smokers, and never-smokers on the modified Stroop task. J Psychopharmacol 17: 310-316.

Paterson NE, Froestl W, Markou A (2005). Repeated administration of the GABAB receptor agonist CG44532 decreased nicotine self-asministration, and acute administration decreased cueinduced reinstatement of nicotine-seeking in rats. Neuropsychopharmacology 30: 119-128.

Patton JH, Stanford MS, Barratt ES (1995). Factor structure of the Barratt impulsiveness scale. J Clin Psychol 51: 768-774.

Paine TA, Slipp LE, Carlezon WA (2011). Schizophrenia-like attentional deficits following blockade of prefrontal cortex $\mathrm{GABA}_{\mathrm{A}}$ receptors. Neuropsychopharmacology 36: 1703-1713.

Pehrson AL, Bondi CO, Totah NKB, Moghaddam B (2013). The influence of NMDA and GABAa receptors and glutamic acid decarboxylase (GAD) activity on attention. Psychopharmacology (Berl) 225: 31-39.

Petty F (1995). GABA and mood disorders: a brief review and hypothesis. J Affect Disord 34: 275-281.

Porrino LJ, Hampson RE, Opris I, Deadwyler SA (2013). Acute cocaine induced deficits in cognitive performance in rhesus macaque monkeys treated with baclofen. Psychopharmacology (Berl) 225: 105-114.

Rolls ET, Loh M, Deco G, Winterer G (2008). Computational models of schizophrenia and dopamine modulation in the prefrontal cortex. Nat Rev Neurosci 9: 696-709.

Shackman AJ, Salomons TV, Slagter HA, Fox AS, Winter JJ, Davidson RJ (2011). The integration of negative affect, pain and cognitive control in the cingulate cortex. Nat Rev Neurosci 12: 154-167.

Shiffman S, Waters AJ (2004). Negative affect and smoking lapses: a prospective analysis. J Consult Clin Psychol 72: 192-201. 
Sommer M, Hajak G, Dohnel K, Meinhardt J, Muller JL (2008). Emotion-dependent modulation of interference processes: an fMRI study. Acta Neurobiol Exp 68: 193-203.

Sofuoglu M, Mouratidis M, Yoo S, Culligan K, Kosten T (2005). Effects of tiagabine in combination with intravenous nicotine in overnight abstinent smokers. Psychopharmacology (Berl) 181: 504-510.

Tiffany ST, Drobes DJ (1991). The development and initial validation of a questionnaire on smoking urges. Br J Addict 86: 1467-1476.

Waters AJ, Shiffman S, Sayette MA, Paty JA, Gwaltney CJ, Balabanis MH (2003). Attentional bias predicts outcome in smoking cessation. Health psychol 22: 378-387.
Watson D, Clark LA, Tellegen A (1988). Development and validation of brief measures of positive and negative affect: the PANAS scales. J Pers Soc Psychol 54: 1063-1070.

Wetherill RR, Jagannathan K, Lohoff W, Ehrman R, O’Brien CP, Childress AR et al (2012). Neural correlates of attentional bias for smoking cues: modulation by variance in the dopamine transporter gene. Addict Biol (e-pub ahead of print).

Winterer G, Weinberger DR (2004). Genes, dopamine and cortical signal-to-noise ratio in schizophrenia. Trends Neurosci 27: 683-690.

World Health Organization (2008): WHO Report on the Global Tobacco Epidemic, 2008. 